\title{
Protoplast Co-culture Bioassay for Allelopathy in Leguminous Plants, Leucaena leucocephala and Mucuna gigantea, Containing Allelochemical Amino Acids, Mimosine and L-DOPA
}

\author{
Daisuke Mori $^{1}$, Shinjiro Ogita ${ }^{2}$, Kouhei Fujise ${ }^{1}$, Aya Inoue ${ }^{1} \&$ Hamako Sasamoto $^{3,4}$ \\ ${ }^{1}$ Graduate School of Environment and Information Sciences, Yokohama National University, Yokohama, Japan \\ ${ }^{2}$ Biotechnology Research Center and Department of Biotechnology, Toyama Prefectural Unversity, Toyama, \\ Japan \\ ${ }^{3}$ Faculty of Environment and Information Sciences, Yokohama National University, Yokohama, Japan \\ ${ }^{4}$ Research Institute for Integrated Science, Kanagawa University, Hiratsuka, Kanagawa, Japan \\ Correspondence: Hamako Sasamoto, Yokohama National University, Yokohama, 240-8501, Japan. E-mail: \\ sasamoto@ynu.ac.jp
}

Received: August 26, 2014 Accepted: September 23, 2014 Online Published: September 26, 2014

doi:10.5539/jps.v4n1p1

URL: http://dx.doi.org/10.5539/jps.v4n1p1

\begin{abstract}
Protoplasts isolated from calluses of two woody leguminous plants, Leucaena leucocephala and Mucuna gigantea, which contain high levels of the allelochemical amino acids, mimosine and L-DOPA, respectively, were co-cultured to examine their allelopathic activities. The young leaves were first confirmed to have strong inhibitory activity by the sandwich method. Callus cultures were induced and sub-cultured from seeds of $L$. leucocephala and from leaves of M. gigantea in Murashige and Skoog's (MS) basal media containing high concentrations of auxin and cytokinins. Protoplasts of both calluses were isolated using the separate well method under strong enzymatic conditions, in high osmotic, $0.8 \mathrm{M}$ mannitol solution. Protoplasts of both species had strong inhibitory effects on the recipient protoplasts of cotyledons of Lactuca sativa (lettuce) co-cultured using 96 multi-well culture plates in the MS basal medium, containing $1 \mu \mathrm{M}$ 2,4-dichlorophenoxyacetic acid, $0.1 \mu \mathrm{M}$ benzyladenine and $0.8 \mathrm{M}$ mannitol solution. Mimosine was less inhibitory than L-DOPA on the growth of lettuce protoplasts at the stage of colony formation. Amino acid profiles of calluses and protoplasts of the above two species and Mucuna pruriens showed very low contents of mimosine and L-DOPA, respectively. The cause of the high inhibitory allelopathic activities found in Leucaena and Mucuna protoplasts on the growth of lettuce protoplasts was discussed.
\end{abstract}

Keywords: allelopathy, amino acids, bioassay methods, callus cultures, Lactuca sativa, Leguminosae, protoplast cultures

\section{Introduction}

The tree leguminous plant, Leucaena leucocephala which grows in tropical and subtropical areas, is a highly invasive species. The strong inhibitory allelopathic activity of leaf litter was studied in vitro by the 'sandwich method' using dried leaves (Fujii \& Hiradate, 2003; Fujii et al., 2003, 2004). Plant tissues contain a large amount of mimosine, which is a toxic non-proteinaceous amino acid (Chou \& Kuo, 1986), and contain another pyridine alkaloid, trigonelline (Ogita et al., 2014). Recently, mimosine was found to have an inhibitory effect on influenza viral neuraminidase (Upadhyay et al., 2012).

Another woody leguminous plant, Mucuna gigantea that grows in the same (sub) tropical areas, was found to show moderate inhibitory allelopathic activity by the sandwich method (Fujii \& Hiradate, 2003). This species is known to contain high amount of an amino acid, L-DOPA, similarly to another herbaceous $M$. pruriens. A variety of the latter species (Hassho-mame) grows in the temperate area. L-DOPA which is biosynthesized from tyrosine, is a strong allelochemical, and is also used for treatment of Parkinson's disease.

To study the mechanism(s) of allelopathy at the cellular level, we developed a new sensitive bioassay method 'protoplast co-culture method' using mixed culture of protoplasts of lettuce and of etiolated leaves of Hassho-mame (Sasamoto et al., 2013). We used the 'protoplast co-culture method' for the suspension cultures of 
mangrove species to clarify the inverse relationship between allelopathic activity and salt tolerance. The allelopathic activities were compared to those measured using the sandwich method. (Hasegawa et al., 2014). The effects of metabolites of nicotinic acid on the growth of lettuce protoplasts were investigated to study the non-inhibitory function of trigonelline (Sasamoto \& Ashihara, 2014).

In the present study, the activity in young leaves of L. leucocephala and M. gigantea was examined by the sandwich method. After callus induction and sub-culture, protoplasts of both species were isolated. Inhibitory effects of L. leucocephala and M. gigantea were investigated in mixed cultures of isolated protoplasts from calluses and protoplasts obtained from lettuce cotyledons at different protoplast densities. In order to elucidate the cause of the strong allelopathic activities of Leucaena and Mucuna protoplasts, the effect of mimosine in medium on the growth of lettuce protoplasts was investigated and compared with that of L-DOPA (Sasamoto et al., 2013).

Furthermore, we investigated the amino acid profile using sub-cultured callus and the protoplasts of $L$. leucocephala, M. gigantea, and M. pruriens. Functions of two amino acids, mimosine and L-DOPA, and related amino acids as possible allelochemicals in protoplasts of Leucaena and Mucuna are also discussed.

\section{Method}

\subsection{Plant Preparation}

Seeds and young leaves of Leucaena leucocephala and Mucuna gigantea were collected on Iriomote island, Okinawa, Japan. Seedlings of M. gigantea were grown in a greenhouse.

Etiolated leaves of M. pruriens (Hassho-mame) and cotyledons of Lactuca sativa (lettuce) were prepared as described previously (Sasamoto et al., 2013). Briefly, seeds of M. pruriens were sterilized with $5 \% \mathrm{NaClO}$ solution for $10 \mathrm{~min}$ were imbibed in tap water. After removing the seed coat, they were planted in autoclaved vermiculite in pots and incubated in a low light condition. Lettuce seeds 'Great Lakes 366' were sterilized with $1.5 \% \mathrm{NaClO}$ solution for $15 \mathrm{~min}$ and washed with autoclaved water three times. They were cultured on $0.8 \%$ agar medium for 1 week to 1 month in a light condition $(16 \mathrm{hr}$ photoperiod, $60 \mu E)$ at $25^{\circ} \mathrm{C}$.

\subsection{Sandwich Method}

Young leaves of $L$. leucocephala and $M$. gigantea were dried at $60{ }^{\circ} \mathrm{C}$ for $18 \mathrm{hrs}$ and stored until use. The procedure of the sandwich method was as described previously (Fujii et al., 2003, 2004). Briefly, 10 and $50 \mathrm{mg}$ dry weight of dried leaves were sandwiched between two layers of $5 \mathrm{ml}$ of $0.5 \%$ agar (powder, gelling temp. $30-31{ }^{\circ} \mathrm{C}$, Nacalai tesque Co. Ltd. Kyoto, Japan) in 6 multi-well plates (Nunc). Length of hypocotyls and roots of germinated seeds of lettuce was measured after 3 days of incubation at $20^{\circ} \mathrm{C}$ in the dark. The control treatment was consisted of seeds germinated in the absence of dried leaves. Data were recorded as \% growth of the control and averaged with standard error (SE).

\subsection{Calluses Induction and Sub-Cultures}

The seeds of $L$. leucocephala were sterilized with $1 \%$ benzalkonium chloride solution for $5 \mathrm{~min}$ and $70 \%$ ethanol for $10 \mathrm{~min}$, then washed with autoclaved water three times. Callus was induced using the Murashige and Skoog's (MS, Murashige \& Skoog, 1962) basal medium containing 3\% sucrose, $30 \mu \mathrm{M}$ of 2,4-dichlorophenoxyacetic acid (2,4-D) and $3 \mu \mathrm{M}$ of thidiazuron, solidified with $0.3 \%$ gellangum.

Callus was induced from the young green leaves of $M$. gigantea. Leaves were sterilized with $1 \% \mathrm{NaClO}$ solution for $10 \mathrm{~min}$ and washed with autoclaved water three times. Cut sections were first planted in $1 \mathrm{~mL}$ liquid MS basal medium containing $3 \%$ sucrose, $10 \mu \mathrm{M}$ each of 2,4-D and benzyladenine (BA), cultured at $100 \mathrm{rpm}$ speed, and proliferated callus was sub-cultured on the same medium composition solidified with $0.8 \%$ agar.

Proliferated calluses were sub-cultured in the dark at 3- to 4-week intervals at $30{ }^{\circ} \mathrm{C}$ ( L. leucocephala), or at 27 ${ }^{\circ} \mathrm{C}(M$. gigantea $)$ and were used for protoplast isolation or stored in a freezer at $-80{ }^{\circ} \mathrm{C}$.

Callus of M. pruriens was obtained from protoplast culture of etiolated leaves using MS medium with $1 \mu \mathrm{M}$ 2,4-D and $5 \mu \mathrm{M} \mathrm{BA}$ as described previously (Sasamoto et al., 2013), and sub-cultured on the same medium composition solidified with $0.8 \%$ agar under the light condition as lettuce seedlings.

\subsection{Protoplast Isolation}

Callus of L. leucocephala after 2-3 weeks of culture was treated for 4-6 hrs with 0.8 M mannitol solution containing 2\% each of Cellulase RS (Yakult Pharmaceutical Ind. Co. Ltd., Tokyo), Driselase 20 (Kyowa Hakko Kogyo), 1\% Funcelase (Yakult) and 0.5\% Pectolyase Y-23 (Kyowa Chemical Products Co. Ltd. Osaka, Japan) in wells of a 24-well culture plate under a sterile condition (the separate well method) (Sasamoto et al., 2013). 
Callus of $M$ gigantea cultured for 1 week was treated in separate wells for 2-3 hrs with $0.8 \mathrm{M}$ mannitol solution containing $1 \%$ each of Cellulase R10, Driselase 20, and $0.5 \%$ Pectolyase Y-23.

Protoplasts of etiolated leaves of M. pruriens were isolated using the separate well method as previously described (Sasamoto et al., 2013). Leaf sections were treated for $18 \mathrm{hrs}$ with $0.4 \mathrm{M}$ mannitol solution containing $1 \%$ each of Cellulase RS, Hemicellulase (H-2125, Sigma) and Macerozyme R-10 (Yakult).

Protoplasts were isolated from lettuce cotyledons in a flask using 1\% each of Cellulase RS and Macerozyme R-10 (Yakult) in $0.8 \mathrm{M}$ mannitol solution with a $20 \mathrm{hr}$-treatment.

After passing through a $42-95 \mu \mathrm{m}$ nylon mesh, protoplasts were purified by washing three times with the mannitol solution followed by centrifugation at $100 \mathrm{~g}$ to $190 \mathrm{~g}(800-1000 \mathrm{rpm})$ for $5 \mathrm{~min}$. All procedures were performed at room temperature.

\subsection{Protoplast Cultures}

Protoplast suspensions in $0.8 \mathrm{M}$ mannitol solution, $5 \mu \mathrm{L}$ each, were put into $50 \mu \mathrm{L}$ of liquid medium in a 96 -well plastic culture plate (Falcon No. 3075). Mucuna or Leucaena protoplasts were cultured with lettuce protoplasts in MS medium containing $1 \mu \mathrm{M}$ of 2,4-D and $0.1 \mu \mathrm{M}$ of BA, 3\% sucrose and $0.8 \mathrm{M}$ mannitol. The protoplast density ranged from $6 \times 10^{2}$ to $10^{5} / \mathrm{mL}$ (L. leucocephala or M. gigantea) and $5 \times 10^{3}$ to $10^{5} / \mathrm{mL}$ (lettuce). Mimosine instead of Leucaena protoplasts was used for culture of lettuce protoplasts where described. After adding $100 \mu \mathrm{L}$ of autoclaved pure water (Milipore Direct-Q UV) to the space between the wells, the plate was tightly sealed with two layers of Parafilm ${ }^{\mathrm{R}}$. The protoplasts were cultured at $28{ }^{\circ} \mathrm{C}$ or at $30^{\circ} \mathrm{C}$ in a humid incubator $\left(\mathrm{CO}_{2}\right.$-incubator without the supply of $\mathrm{CO}_{2}$, APC-30DR, ASTEC Co. Ltd.). Numbers of enlarged- and divided- protoplasts were counted at 4 to 9 days of culture under an inverted microscope (Olympus CK40 or IX71).

Growth of lettuce protoplasts was described as the numbers of non-spherically enlarged ( $>50 \mu \mathrm{m}$ diameter) and divided protoplasts or colonies composed of more than 4 cells in a well. All data were described as the percentage of the control without Leucaena or Mucnuna protoplasts or mimosine at each density of lettuce protoplasts. Averages with SE were calculated from the values at different densities of lettuce protoplast as described previously (Sasamoto \& Ashihara, 2014; Hasegawa et al., 2014).

\subsection{Amino Acid Analysis}

Amino acids were extracted and analyzed from calluses and protoplasts as previously reported (Ogita et al., 2014; Tsuchiya et al., 2013). Briefly, after extraction with $80 \%$ ethanol at $60^{\circ} \mathrm{C}$ three times, combined fractions were evaporated to dryness using a Vacuum centrifugal evaporator (CVE-3100, EYELA, Tokyo Japan) with a glass cold trap (Uni trap UT-1000, EYELA). The residues were dissolved in $50 \mathrm{mM}$ borate buffer (pH8.0, with 0.05 mM EDTA). Amino acids were precolumn-derivatized with 4 - fluoro -7 - nitrobenzo $-s-$ oxa $-1,3$ - diazole (NBD-F) (Kotaniguchi \& Kawakatsu, 1987) and analyzed using a gradient HPLC system (Gilson 305 system) at $30{ }^{\circ} \mathrm{C}$. Column was YMC-Pack ODS-A reversed-phase column $(4.6 \mathrm{~mm} \times 150 \mathrm{~mm})$. The flow rate was $1.0 \mathrm{~mL} /$ min. Fluorescence was measured at ex. $470 \mathrm{~nm}$ and em. $540 \mathrm{~nm}$. Mimosine was detected with the standard as for the plant tissues (Ogita et al., 2014). Data were calculated from three different concentrations for each sample.

The retention times of unidentified amino acid $\left(\mathrm{u}^{*}\right)$, mimosine and L-DOPA in this system were $23.9 \mathrm{~min}, 25.5$ min, and $38.1 \mathrm{~min}$, respectively. The content of the unidentified amino acid was described as glycine equivalent.

\section{Results and Discussion}

\subsection{Sandwich Method: Strong Inhibitory Activities of Young Leaves of L. leucocephala and M. gigantea}

Though, some allelochemicals of secondary metabolites, might accumulate during the late growth stage of plants, higher inhibitory activity was reported in the young green leaves of soybean using the sandwich method (Sasamoto et al., 2012) compared to that of leaf litter. Strong inhibitory allelopathic activities of leaf litter of $L$. leucocephala and moderate activity of leaf fall of $M$. gigantea were obtained using the sandwich method (Fujii $\&$ Hiradate, 2003). In this report, we applied the sandwich method to the young leaves of L. leucocephala and $M$. gigantea species. As shown in Figure 1a (L. leucocephala) and 1b (M. gigantea), young leaves of both species had very strong inhibitory activities on root elongation of lettuce. M. gigantea had lower activity than $L$. leucocephala on elongation of lettuce hypocotyls. These findings were consistent with the report on $L$. leucocephala showing higher allelopathic activity and higher content of mimosine in young leaves than in mature tissues (Chou \& Kuo, 1986; Xuan et al., 2006). 

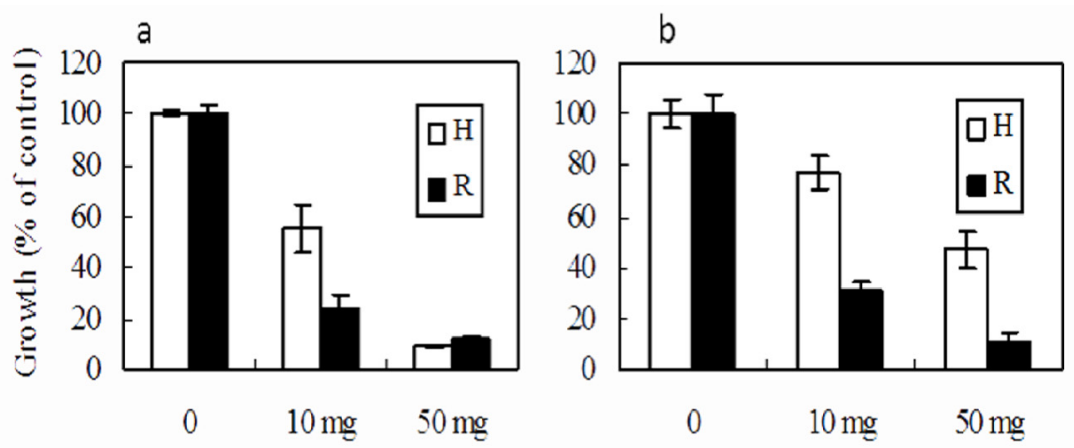

Figure 1. Bioassay of allelopathy by the sandwich method of young leaves of $L$. leucocephala (a) and $M$. gigantea (b). Data were elongation percentages of control with SE of lettuce hypocotyl (H) and root (R)

\subsection{Hormonal Conditions for Calluses Induction and Proliferation}

Calluses were induced from the hypocotyl of L. leucocephala seedlings on MS medium containing high concentrations of plant growth regulators; $30 \mu \mathrm{M}$ of an auxin, 2,4-D and $3 \mu \mathrm{M}$ of thidiazuron, which is a strong cytokinin. The same combination of plant hormones was successful for sub-culture. These concentrations of 2,4-D and thidiazuron are very high and strong even compared with those of the subculture medium for $M$. gigantea, which was $10 \mu \mathrm{M}$ each of 2,4-D and a weak cytokinin, BA. The same hormonal condition in the sub-culture medium was reported for leaf callus cultures of other tree (Derris indica) and woody (Caesalpinia crista) leguminous plants (Inoue et al., 2010). A herbaceous leguminous plant, e.g., M. pruriens, also preferred high cytokinin condition which was $1 \mu \mathrm{M}$ of 2,4-D and $5 \mu \mathrm{M}$ of BA for callus proliferation (Sasamoto et al., 2013).

\subsection{Strong Enzymatic Conditions for Protoplast Isolation of L. leucocephala and M. gigantea}

In our previous report, for protoplast isolation from etiolated leaves of $M$. pruriens, which contains a large amount of the allelochemical, L-DOPA, the enzymatic condition; $1 \%$ each of Cellulase RS, Hemicellulase and Macerozyme in $0.4 \mathrm{M}$ mannitol, was required in the separate well method using 24-well culture plate (Sasamoto et al., 2013). In this report, the same separate well method, which might reduce the formation of oxidized substances, was required for isolation of protoplasts from calluses of both L. leucocephala and M. gigantea. In addition, a higher osmotic condition $(0.8 \mathrm{M}$ mannitol) and strong enzymatic conditions were required as follows. For the callus of $L$. leucocephala, high concentrations of strong cell wall degrading enzymes; $2 \%$ each of Cellulase RS, Driselase 20 and $0.5 \%$ Pectolyase Y-23 and addition of $1 \%$ Funcellase, were all effective for isolation of protoplasts.

Callus of M. gigantea cultured for one week was used for viable protoplasts isolation. Protoplasts were isolated after 2-3 hrs incubation in $0.8 \mathrm{M}$ mannitol solution containing $1 \%$ Cellulase R10, $1 \%$ Driselase 20, and 0.5\% Pectolyase Y-23. Callus cultured for more than 10 days appeared brown and the efficiency of protoplast isolation was reduced. A dim red color, which might be dopaquinone, was observed in the protoplast suspension.

In the present study, lettuce protoplasts were isolated, for protoplast co-culture with L. leucocephala or M. gigantea, in 1\% each of Cellulase RS and Macerozyme in $0.8 \mathrm{M}$ mannitol solution instead of $0.4 \mathrm{M}$ mannitol solution with M. pruriens (Sasamoto et al., 2013).

Figure 2 shows the isolated protoplasts of lettuce, L. leucocephala and M. gigantea. The diameters of them are around 30-50 $\mu \mathrm{m}$. Leucaena protoplasts from the callus contained many starch granules which were stained with $\mathrm{I}_{2}$-KI solution (data not shown), while lettuce protoplasts contain green chloroplasts. 


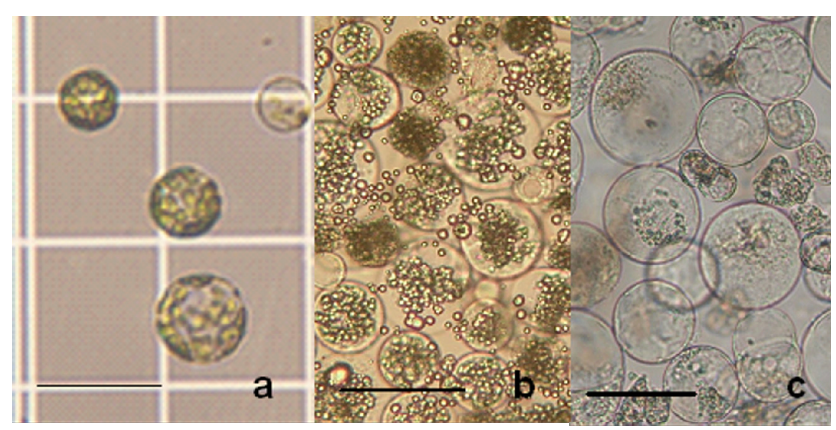

Figure 2. Isolated protoplasts of lettuce (a), L. leucocephala (b) and M. gigantea (c). Bar $=50 \mu \mathrm{m}$

\subsection{Protoplast Co-culture Method: Strong Inhibitory Activities of L. leucocephala and M. gigantea on lettuce}

As shown in Figure 3, Leucaena protoplasts inhibited the growth of lettuce protoplasts depending on the density. L. leucocephala at $2 \times 10^{4} / \mathrm{mL}$ completely inhibited the growth of lettuce protoplasts. $50 \%$ inhibition was obtained with only $10^{3} / \mathrm{mL}$ (50 protoplasts / $50 \mu \mathrm{L}$ medium). This is the $1 / 100$ of the effective density of $M$. pruriens protoplasts which inhibited the growth of lettuce protoplasts (Sasamoto et al., 2013). Similarly, as shown in Figure 4, M. gigantea protoplasts at low protoplast density inhibited the growth of lettuce protoplasts. A protoplast density of $2 \times 10^{4} / \mathrm{mL}$ completely inhibited the growth of lettuce protoplasts.

The combination of plant growth regulators in the medium, $1 \mu \mathrm{M}$ of 2,4-D and $0.1 \mu \mathrm{M}$ of BA, was optimum for growth of lettuce protoplasts (Sasamoto et al., 2013), but protoplasts of Leucaena or M. gigantea did not divide under the same condition (data not shown). In contrast to M. pruriens, which stimulated the growth of lettuce protoplasts at a low protoplast density (Sasamoto et al., 2013), no stimulating effect of M. gigantea was observed at a low protoplast density.

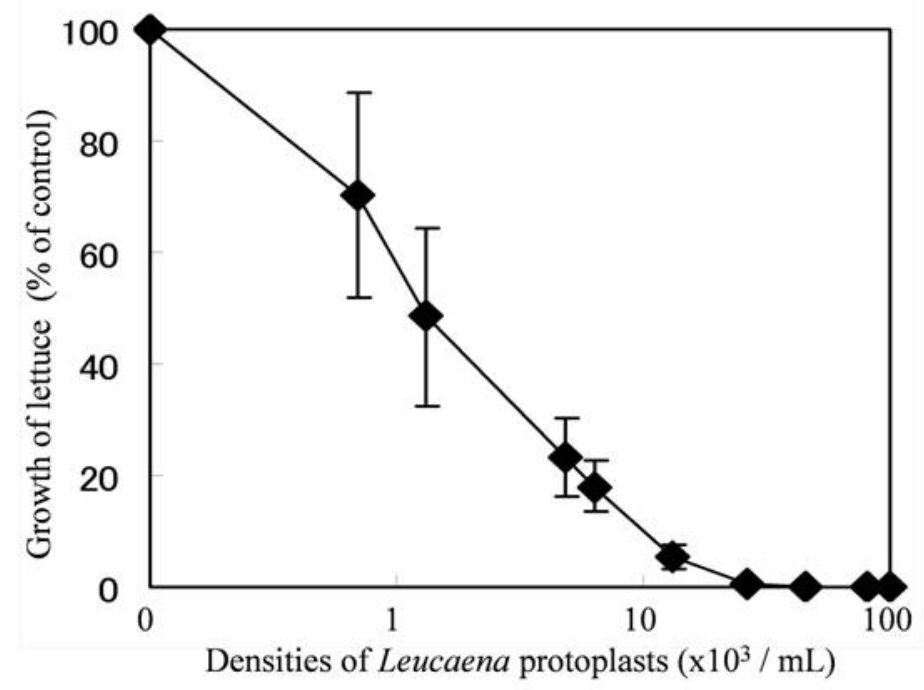

Figure 3. Effects of $L$. leucocephala protoplasts on the growth of lettuce protoplasts co-cultured for 4 days in the

MS basal medium containing $1 \mu \mathrm{M} 2,4-\mathrm{D}$ and $0.1 \mu \mathrm{M}$ BA, 3\% sucrose and $0.8 \mathrm{M}$ mannitol. Numbers of enlarged or divided protoplasts were counted and described as the $\%$ of control without Leucaena protoplasts. Data are averages with SE at $5-200 \times 10^{3} / \mathrm{mL}$ lettuce protoplast density 


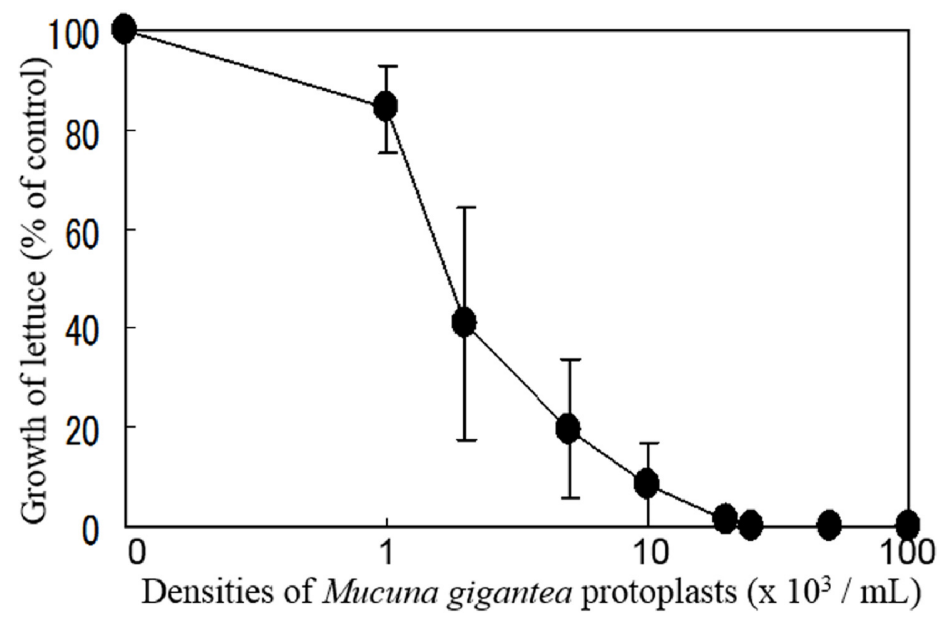

Figure 4. Effects of $M$. gigantea protoplasts on protoplast cultures of lettuce after 4-6 days of culture. Medium was the same as in Figure 3. Numbers of enlarged or divided protoplasts were counted and described as the \% of control without Mucuna protoplasts. Data are averages with SE at 5-100 $\times 10^{3} / \mathrm{mL}$ lettuce protoplast density

Percentage inhibition of growth to the control without L. leucocephala or Mucuna protoplasts, was averaged at different protoplast densities of lettuce. This method was useful for comparison of effects of three different metabolites (Sasamoto \& Ashihara, 2014) and among different mangrove species (Hasegawa et al., 2014).

\subsection{Effects of Mimosine on Lettuce Protoplasts}

The high mimosine content of young leaves of L. leucocephala has been reported to be the cause of their high allelopathic activity (Xuan et al., 2006). We examined the effect of mimosine on protoplast cultures of lettuce. As shown in Figure 5, at day 4, enlargement of lettuce protoplasts was partly inhibited by $300 \mu \mathrm{M}$ and $1 \mathrm{mM}$ mimosine supplied in the medium. At 9 days of culture, colony formation was almost completely inhibited at 300 $\mu \mathrm{M}$ of mimosine, which corresponds to the inhibition by $2 \times 10^{4} / \mathrm{mL}$ of the Leucaena protoplasts in co-culture (Figure 3). The mimosine content in protoplasts of L. leucocephala to inhibit the growth of lettuce protoplasts was calculated to be $150 \mu$ moles / $10^{7}$ protoplasts.

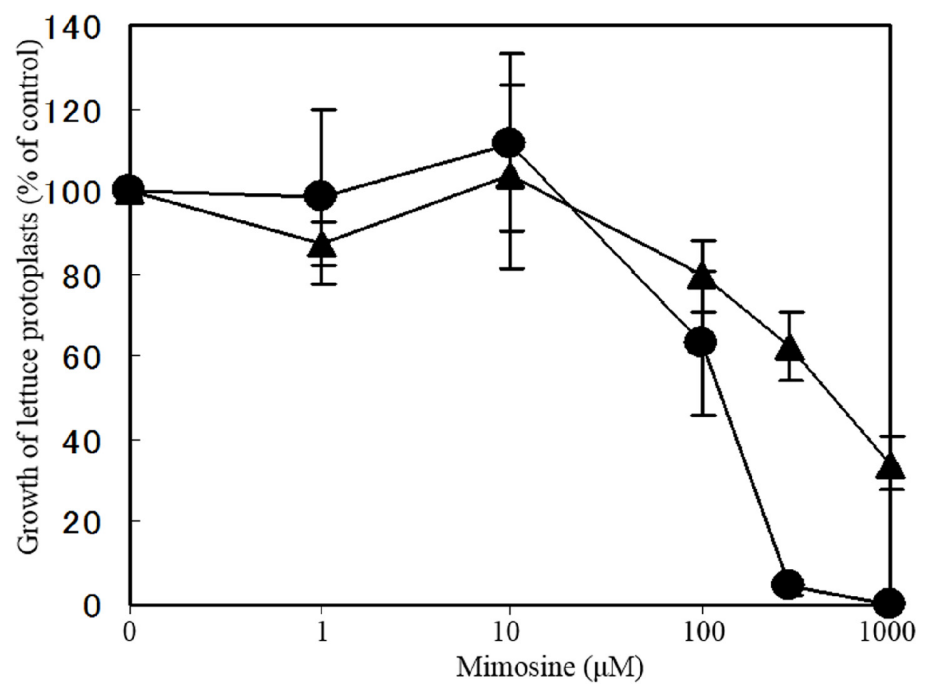

Figure 5. Effects of minosine on growth of lettuce protoplasts. Numbers of enlarged and divided protoplasts, and colonies after 4 days of culture (triangle) and after 9 days of culture (circle), were counted and described as the $\%$ of control. Medium was the same as in Figure 3 . Data are averages with SE at $5 \times 10^{3}-10^{5} / \mathrm{mL}$ lettuce protoplast density 


\subsection{Amino Acid Profiles of Calluses and Protoplasts}

The amino acid composition of protoplasts was compared with that of calluses. Amino acid profiles of Leucaena callus and protoplasts are shown in Table 1 . The callus contained glutamic acid ( $11 \mu$ moles $/ \mathrm{g}$ fresh weight $)$ and glutamine $(8.9 \mu$ moles / g fresh weight) as major amino acids, and a large amount of unidentified amino acid near the peak of alanine. Mimosine content was low $0.1 \mu$ moles / g fresh weight. Protoplasts contained an extremely high content $(76.5 \%)$ of glutamine, but a low content of mimosine, $0.11 \mu$ moles $/ 10^{7}$ protoplasts, which was similar to their contents in callus. Both callus and protoplasts contained mimosine only $0.2 \%$ of total amino acids, but they contained different contents of the major amino acids. Such a difference in contents of amino acids between callus and their protoplasts had been reported in several tree mangrove species, which required strong enzymatic conditions for protoplast isolation (Tsuchiya et al., 2013). For isolation of Leucaena protoplasts, very strong enzymatic conditions including Fancellase were needed.

Table 1. Amino acid contents of callus and protoplasts of Leucaena leucocephala

\begin{tabular}{lrlrl}
\hline & \multicolumn{2}{c}{ Callus } & \multicolumn{2}{c}{ Protoplasts } \\
& nmoles/g fresh weight & nmoles/10 & protoplasts \\
\hline His & 919 & $(1.8)$ & 325 & $(0.6)$ \\
Arg & 500 & $(1.0)$ & 410 & $(0.8)$ \\
Asn & 1977 & $(4.0)$ & 289 & $(0.5)$ \\
Gln & 8861 & $(17.8)$ & 40554 & $(76.5)$ \\
Ser & 4028 & $(8.1)$ & 684 & $(1.3)$ \\
Asp & 617 & $(1.2)$ & 4 & $(0.0)$ \\
Gly & 1068 & $(2.1)$ & 68 & $(0.1)$ \\
Glu & 10956 & $(22.0)$ & 4076 & $(7.7)$ \\
Thr & 2037 & $(4.1)$ & 165 & $(0.3)$ \\
Ala & 1540 & $(3.1)$ & 969 & $(1.8)$ \\
u* & 10588 & $(21.2)$ & 2947 & $(5.6)$ \\
Pro & 2034 & $(4.1)$ & 236 & $(0.4)$ \\
L-Mimosine & 101 & $(0.2)$ & 110 & $(0.2)$ \\
Met & 138 & $(0.3)$ & 207 & $(0.4)$ \\
Val & 1234 & $(2.5)$ & 383 & $(0.7)$ \\
(Cys)2 & 14 & $(0.0)$ & 42 & $(0.1)$ \\
Lys & 179 & $(0.4)$ & 274 & $(0.5)$ \\
Phe & 1642 & $(3.3)$ & 549 & $(1.0)$ \\
Ile & 213 & $(0.4)$ & 126 & $(0.2)$ \\
Leu & 1012 & $(2.0)$ & 539 & $(1.0)$ \\
Tyr & 240 & $(0.5)$ & 74 & $(0.1)$ \\
total & 49898 & & 53029 & \\
\hline
\end{tabular}

$\mathrm{u}^{*}$ : unidentified amino acid (nmoles glycine eq.).

Values in parentheses are Percentages.

The callus and protoplasts of L. leucocephala had amino acid profiles totally different from those of Leucaena plant tissues, e.g. leaves, flowers and seeds (Ogita et al., 2014), which were used to examine the relation between major amino acids, mimosine and asparagine. The leaves had a mimosine content of 20-40 $\mu$ moles / g fresh weight, which was 400-800 times that in the callus. Callus often have a lower content of metabolites than the original plant tissues (Sasamoto et al., 2013). As the volume of a spherical protoplast is $14 \mathrm{pL}$ (30 $\mu \mathrm{m}$ diameter) and $65 \mathrm{pL}(50 \mu \mathrm{m}$ diameter), the concentration of mimosine in Leucaena protoplasts was calculated to be 0.78 
$\mathrm{mM}$ to $0.17 \mathrm{mM}$. These mimosine concentrations were sufficient for inhibition of growth in lettuce protoplast culture (Figure 5).

Amino acid profiles of $M$. gigantea callus and protoplasts are shown in Table 2. Callus contained alanine (1.5 $\mu$ moles / g fresh weight), serine $(0.6 \mu$ moles / $\mathrm{g}$ fresh weight $)$ and glutamine $(0.6 \mu$ moles / $\mathrm{g}$ fresh weight $)$ as major amino acids. In contrast, alanine (15.8\%) and an unidentified amino acid (23.6\%) were the major amino acids in the protoplasts. L-DOPA was not detected in this species, though a high content of tyrosine, the precursor of L-DOPA biosynthesis, was detected in both the callus (7.6\%) and protoplasts (5.8\%). The content of the allelochemical L-DOPA was also lower in callus cultures than in the original plant tissues (Sasamoto et al., 2013).

Table 2. Amino acid contents of callus and protoplasts of Mucuna gigantea

\begin{tabular}{|c|c|c|c|c|}
\hline \multirow[b]{2}{*}{ His } & \multicolumn{2}{|c|}{$\begin{array}{c}\text { Callus } \\
\text { nmoles/g fresh weight }\end{array}$} & \multicolumn{2}{|c|}{$\begin{array}{c}\text { Protoplasts } \\
\text { nmoles } / 10^{7} \text { protoplast }\end{array}$} \\
\hline & 33 & (0.9) & 356 & $(2.2)$ \\
\hline Arg & 34 & $(1.0)$ & 597 & (3.6) \\
\hline Asn & 29 & $(0.8)$ & 320 & (1.9) \\
\hline Gln & 555 & (16.1) & 838 & $(5.1)$ \\
\hline Ser & 580 & (16.8) & 1258 & (7.6) \\
\hline Asp & 3 & $(0.1)$ & 70 & $(0.4)$ \\
\hline Gly & 12 & $(0.4)$ & 345 & $(2.1)$ \\
\hline Glu & 69 & $(2.0)$ & 148 & $(0.9)$ \\
\hline Thr & 41 & (1.2) & 516 & (3.1) \\
\hline Ala & 1455 & $(42.1)$ & 2612 & $(15.8)$ \\
\hline $\mathrm{u}^{*}$ & 41 & $(1.2)$ & 3896 & $(23.6)$ \\
\hline Pro & 63 & (1.8) & 780 & $(4.7)$ \\
\hline Met & 33 & $(0.9)$ & 189 & (1.1) \\
\hline Val & 90 & $(2.6)$ & 974 & (5.9) \\
\hline (Cys) 2 & 13 & $(0.4)$ & - & \\
\hline Lys & 33 & $(1.0)$ & 741 & $(4.5)$ \\
\hline Phe & 36 & $(1.0)$ & 503 & (3.1) \\
\hline Ile & 24 & $(0.7)$ & 544 & (3.3) \\
\hline Leu & 45 & (1.3) & 848 & $(5.1)$ \\
\hline L-DOPA & - & & - & \\
\hline Tyr & 263 & (7.6) & 957 & $(5.8)$ \\
\hline total & 3453 & & 16493 & \\
\hline
\end{tabular}

$\mathrm{u}^{*}$ : unidentified amino acid (nmoles glycine eq.)

-: not detected. Values in parentheses are percentages.

Table 3 shows the amino acid profile of M. pruriens callus and protoplasts. Protoplasts isolated from etiolated shoots had a high content of asparagine and alanine. Callus had a high content of alanine $(18.5 \mu$ moles $/ \mathrm{g}$ fresh weight), serine ( $4.3 \mu$ moles / $\mathrm{g}$ fresh weight $)$ and glutamine $(3.8 \mu$ moles / $\mathrm{g}$ fresh weight $)$ as major amino acids. High alanine and serine contents were similar to that of $M$. gigantea callus (Table 2). L-DOPA content was high in protoplasts $(3.5 \%)$, and low in the callus $(0.1 \%)$, while the tyrosine content was lower in protoplasts. The concentration of L-DOPA in M. pruriens protoplasts with a diameter of $20 \mu \mathrm{m}(4.2 \mathrm{pL})$ was calculated to be 5.2 $\mathrm{mM}$. In contrast to M. gigantea protoplasts and callus, M. pruriens protoplasts had a high L-DOPA content and low tyrosine content. 
The protoplasts of $M$. gigantea and callus of L. leucocephala had a high content of an unidentified amino acid, which was not detected in $M$. pruriens. These results suggest no common correlation between the contents and strong allelopathic activities of protoplasts of these three species.

Table 3. Amino acid contents of callus and protoplasts of etiolated leaves of Mucuna pruriens

\begin{tabular}{lrlrl}
\hline & \multicolumn{2}{c}{ Callus } & \multicolumn{2}{c}{ Protoplasts } \\
& nmoles/g fresh weight & nmoles/107 & protoplasts \\
\hline His & 1081 & $(3.0)$ & 169 & $(2.8)$ \\
Arg & 435 & $(1.2)$ & 276 & $(4.5)$ \\
Asn & 1198 & $(3.3)$ & 2954 & $(48.3)$ \\
Gln & 3800 & $(10.5)$ & 259 & $(4.2)$ \\
Ser & 4323 & $(12.0)$ & 253 & $(4.1)$ \\
Asp & 639 & $(1.8)$ & 42 & $(0.7)$ \\
Gly & 735 & $(2.0)$ & 74 & $(1.2)$ \\
Glu & 1360 & $(3.8)$ & 54 & $(0.9)$ \\
Thr & 12 & $(0.0)$ & 143 & $(2.3)$ \\
Ala & 18494 & $(51.2)$ & 723 & $(11.8)$ \\
u* & - & & - & \\
Pro & 921 & $(2.5)$ & 99 & $(1.6)$ \\
Met & 167 & $(0.5)$ & 31 & $(0.5)$ \\
Val & 1091 & $(3.0)$ & 216 & $(3.5)$ \\
(Cys)2 & 35 & $(0.1)$ & 22 & $(0.4)$ \\
Lys & 102 & $(0.3)$ & 189 & $(3.1)$ \\
Phe & 247 & $(0.7)$ & 92 & $(1.5)$ \\
Ile & 440 & $(1.2)$ & 121 & $(2.0)$ \\
Leu & 383 & $(1.1)$ & 170 & $(2.8)$ \\
L-DOPA & 36 & $(0.1)$ & 216 & $(3.5)$ \\
Tyr & 641 & $(1.8)$ & 13 & $(0.2)$ \\
total & 36140 & & 6116 & \\
\hline & & & &
\end{tabular}

$\mathrm{u}^{*}$ : unidentified amino acid (nmoles glycine eq.).

-: not detected. Values in parentheses are percentages.

\subsection{Cause of Strong Allelopathic Activity in Protoplasts}

The high inhibitory activity of young leaves of L. leucocephala was confirmed by the sandwich method (Figure 1a, section 3.1). The protoplasts also showed similar high inhibitory activity by protoplast co-culture method using callus cultures of L. leucocephala (Figure 3, section 3.4).

Leucaena plant tissues contain a large amount of pyridine alkaloids, mimosine and trigonelline (Ogita et al., 2014). However, as trigonelline did not inhibit the growth of lettuce protoplasts, it has been discussed as a detoxification product (Sasamoto \& Ashihara, 2014). Non-proteinaceous amino acid, mimosine is thought to be the main allelochemical in Leucaena plant (Xuan et al., 2006). Here we examined the direct effect of mimosine in the medium, and found that it inhibited the growth of lettuce protoplasts (Figure 5). However, mimosine was inhibitory only at high concentrations $(100-300 \mu \mathrm{M})$. As $50 \mu \mathrm{M}$ of L-DOPA inhibited the growth of lettuce protoplasts completely (Sasamoto et al., 2013), 6 times higher concentration of mimosine in the medium was needed for inhibition. The value, $150 \mu$ moles $/ 10^{7}$ protoplasts, calculated (section 3.5 ), is much higher than the experimental result (Table 1). Considering the low content of mimosine in callus and protoplasts $(1 / 200-1 / 400$ 
of leaf tissues, section 3.6), the strong inhibitory activity of Leucaena protoplasts co-cultured with lettuce protoplasts, did not correlate directly with the low mimosine content in isolated protoplasts.

Using the sandwich method, young leaves of $M$. gigantea with high inhibitory activity showed strong inhibitory allelopathic activities (Figure 1b). Similar high inhibitory activity of protoplasts was found in the 'protoplast co-culture method', developed using callus culture of $M$. gigantea (Figure 4, section 3.4).

Plants of M. gigantea contain L-DOPA as plants of M. pruriens. L-DOPA at $50 \mu \mathrm{M}$ totally inhibited growth of lettuce protoplasts, while no effect on rice protoplasts was obtained. Such phenomena were similar to those of field test of allelopathy (Sasamoto et al., 2013). As the same strong inhibition was obtained with M. gigantea by the sandwich method and protoplast method, the mechanism is assumed to be the same. L-DOPA was detected in M. pruriens protoplasts (Table 3), but not in M. gigantea protoplasts while the precursor tyrosine content was high in both protoplasts and callus (Table 2). The strong inhibitory activities of two Mucuna species could not be explained by the low L-DOPA contents of freshly isolated protoplasts detected by amino acid analysis. Concentrations of each putative allelochemical in each protoplast were calculated to be 0.17 to $0.78 \mathrm{mM}$ mimosine in L. leucocephala, $5.2 \mathrm{mM} \mathrm{L-DOPA} \mathrm{in} \mathrm{M.} \mathrm{pruriens.} \mathrm{These} \mathrm{values} \mathrm{are} \mathrm{high} \mathrm{enough} \mathrm{to} \mathrm{inhibit} \mathrm{the}$ growth of lettuce protoplasts. M. gigantea protoplasts were calculated to have a tyrosine content of 1.5 to 6.7 $\mathrm{mM}$.

There are several possible explanations for this discrepancy. (1) Leucaena or Mucuna protoplasts quickly synthesize mimosine or L-DOPA during culture under non-optimal hormonal conditions in a high osmotic solution. (2) Secondary effects of low contents of mimosine or L-DOPA, during several hrs of treatment for isolation of protoplasts, or cell death during culture may cause a different allelopathic effect during culture. (3) The chemicals dissolved in the medium were not efficiently incorporated into lettuce protoplasts, but cell to cell interaction might accelerate the process; (4) Some specific cellular mechanism(s) can affect lettuce protoplasts through only small amount of chemicals.

Therefore, this protoplast co-culture method using L. leucocephala and M. gigantea calluses may be useful as a sensitive bioassay of allelopathy. The inhibitory activities of protoplasts on the growth of lettuce protoplasts could be quantitated using a small number (50-5000/50 $\mu \mathrm{L})$. Investigation of putative allelochemicals in recipient lettuce protoplast cultures will help to elucidate the cellular mechanism underlying the allelopathic activity. Furthermore, the method can be applied on different recipient plant species under different culture conditions, e.g., high and low temperature or different nutritional conditions, in order to simulate the possible environmental risk of invasiveness of these species (Sasamoto et al., 2013).

\section{References}

Chou, C. H., \& Kuo, Y. L. (1986). Allelopathic research of subtropical vegetation in Taiwan III. Allelopathic exclusion of understory by Leucaena leucocephala (Lam.) de Wit. Journal Chemical Ecology, 12(6), 1431-1448. http://dx.doi.org/10.1007/BF01012362

Fujii, Y., \& Hiradate, S. (Eds.). (2003). Research achievement of allelopathy laboratory in NIAES, 1983 to 2001 (Part 2, p. 982. p. 1060. p. 1180).

Fujii, Y., Parvez, S. S., Parvez, M. M., Ohmae, Y., \& Iida, O. (2003). Screening of 239 medicinal plant species for allelopathic activity using the sandwich method. Weed Biology and Management, 3, 233-241. http://dx.doi.org/10.1046/j.1444-6162.2003.00111.x

Fujii, Y., Shibuya, T., Nakatani, K., Itani, T., Hiradate, S., \& Parvez, M. M. (2004). Assesment method for allelopathic effect from leaf litter leachates. Weed Biology and Management, 4, 19-23. http://dx.doi.org/10.1111/j.1445-6664.2003.00113.x

Hasegawa, A., Oyanagi, T., Minagawa, R., Fujii, Y., \& Sasamoto, H. (2014). An inverse relationship between allelopathic activity and salt tolerance in suspension cultures of three mangrove species, Sonneratia alba, $S$. caseolaris and S. ovata: Development of a bioassay method for allelopathy, the protoplast co-culture method. Journal Plant Research,

Inoue, A., Tsuchiya, S., Minagawa, R., \& Sasamoto, H. (2010). Protoplast isolation and cultures of three leguminous mangrove plants. Proceedings of the $74^{\text {th }}$ Annual Meeting of the Botanical Society of Japan, $\mathrm{p}$. 234.

Kotaniguchi, H., \& Kawakatsu, M. (1987). Automatic amino acid analysis utilizing 4-fluoro-7-nitrobenzos-oxa-diazole. Journal of Chromtography, 420, 141-145. http://dx.doi.org/10.1016/0378-4347(87)80164-0 
Murashige, T., \& Skoog, F. (1962). A revised medium for rapid growth and bioassay with tobacco tissue cultures. Physiologia Plantarum, 15, 473-497. http://dx.doi.org/10.1111/j.1399-3054.1962.tb08052.x

Ogita, S., Kato, M., Watanabe, S., \& Ashihara, H. (2014). The Co-occurrence of two pyridine alkaloids, mimosine and trigonelline, in Leucaena leucocephala. Zeitschrift fur Naturforschung, 69c, 124-132. http://dx.doi.org/10.5560/ZNC.2013-0137

Sasamoto, H., \& Ashihara, H. (2014). Effect of nicotinic acid, nicotinamide and trigonelline on the proliferation of lettuce cells derived from protoplasts. Phytochemistry Letters, 7, 38-41. http://dx.doi.org/10.1016/j.phytol.2013.09.008

Sasamoto, H., Fujise, K., Kobayashi, Y., Mori, D., Oyanagi, T., \& Hasegawa, A. (2012). Development of 'Protoplast method' for bioassay of allelopathy in plants. Proceedings of the 76th Annual Meeting of the Botanical Society of Japan, p. 128.

Sasamoto, H., Murashige-Baba, T., Inoue, A., Sato, T., Hayashi, S., \& Hasegawa, A. (2013). Development of a new method for bioassay of allelopathy using protoplasts of a leguminous plant, Mucuna pruriens with a high content $\mathrm{pf}$ the allelochemical L-DOPA. Journal of Plant Studies, 2(2), 71-80. http://dx.doi.org/10.5539/jps.v2n2p71

Tsuchiya, S., Ogita, S., Kawana, Y., Oyanagi, T., Hasegawa, A, \& Sasamoto, H. (2013). Relation between amino acids profiles and recalcitrancy of cell growth or salt tolerance in tissue and protoplast cultures of three mangrove species, Avicennia alba, Bruguiera sexangula, and Sonneratia alba. American Journal of Plant Sciences, 4(7), 1366-1374. http://dx.doi.org/10.4236/ajps.2013.47167

Xuan, T. D., Elzaawely, A. A., Deba, F., Fukuta, M., \& Tawata, S. (2006). Mimosine in Leucaena as a potent bio-herbicide. Agronomy for Sustainable Development, 26, 89-97. http://dx.doi.org/10.1051/agro:2006001

Upadhyay, A., Chompoo, J., Taira, N., Fukuta, M., Gima, S., \& Tawata, S. (2011). Solid-phase synthesis of mimosine tetrapeptides and their inhibitory activities on neuraminidase and tyrosinase. Journal of Agricultural and Food Chemistry, 59(24), 12858-12863. http://dx.doi.org/10.1021/jf203494t

\section{Copyrights}

Copyright for this article is retained by the author(s), with first publication rights granted to the journal.

This is an open-access article distributed under the terms and conditions of the Creative Commons Attribution license (http://creativecommons.org/licenses/by/3.0/). 